Zhu Peng, Guo-yue Yang

\title{
Liang-shu Lu: a Brief Biography
}

\author{
Liang-shu Lu \\ Professor-Engineer
}

Liang-shu Lu was born in Shanghai in November 1924. In January 1947, he graduated from the School of Agriculture of Nanking University. Lu, an expert in wheat breeding and cultivation, is also an influential scientist in agriculture of China. In 1994, Lu was elected a member to the Chinese Academy of Engineering.

$\mathrm{Lu}$ once worked as the president of the Chinese Academy of Agricultural Sciences (CAAS), the vice president of the Chinese Academy of Engineering (CAE), the vice chairman of the Science and Technology Committee of the Ministry of Agriculture of China, and the president of the Chinese Society of Agricultural Sciences (CSAS). Besides, he was a delegate to the third and the

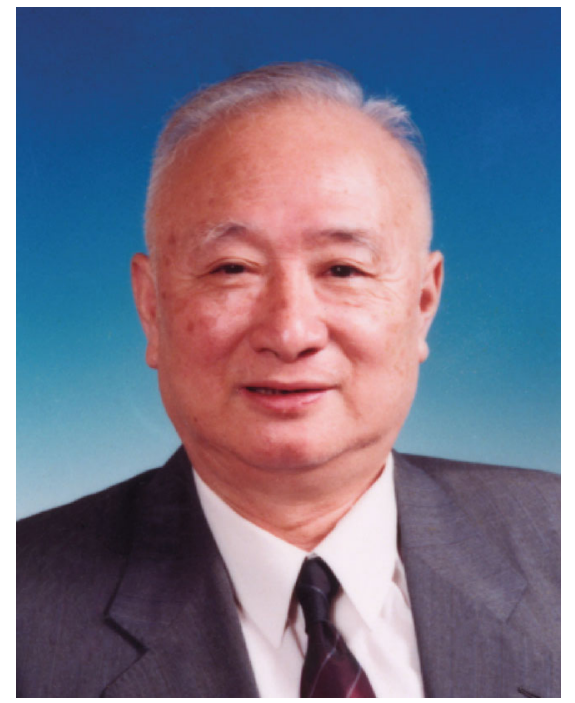
fifth National People's Congress (NPC). Now, he serves as the chairman of the Advisory Group of the Agricultural Experts of China, the chairman of the National Consultant Committee on Food and Nutrition, honorary president of CSAS, and the honorary chairman of the academic board for the CAAS.

Lu has been devoting himself to the promotion of wheat breeding and cultivation for many years, and has been making great contributions over the past years to the advancement of agriculture in China.

Lu spent much of his time on consultant study for policy-making and strategy-development, concerning such issues as the reform of the science and technology system of China, the medium and long-term development of science and technology of China, and the development of food and nutrition in China from 2001 to 2010. He exploited new domains that improved the food structure and its development, and proposed many important and creative new ideas such as changing the traditional concepts of food into the modern ones, promoting plantation in tripartite-structure, and developing modern intensive and sustainable agriculture.

Lu chaired many important research projects, including the great priority project funded by the National Foundation of Natural Sciences, Research on the Medium and Long-term Food Development Strategy of China, which was a systematic research of the food industry in China, focusing on the following 6 aspects: the level of nutrition, the food production, the food distribution, the food consumption, the material investment in the food industry, and the progress of technology and agricultural development in different areas. The results of the great priority project were adopted by consultant institutions and decision-making departments of the government, providing scientific grounds for the making of The 1990s National

Manuscript received February 20, 2014; accepted October 30, 2015

Zhu Peng ( $ه)$

School of Public Administration, Academy of Education Studies, Central South University, Changsha 410083, China

Email: 312518075@qq.com 
Guidelines for Reform and Development of Food Structures, and winning Lu and his team a first prize of the Ministry of Agriculture Prizes for Scientific and Technological Progress, and a second prize of the National Prizes for Scientific and Technological Progress.

Lu produced many creative strategic ideas as part of the fruits of other important research projects concerning agriculture. Such ideas as "intensive and sustainable modern agriculture," "food safety" and "three-element structure of plantation," exercised important influences in the decision-making of the top-level authorities or institutions.

Lu presided over several important wheat breeding and cultivation projects, developing many fine varieties of wheat, including the "East China 6" series which is early ripe, resistant to rust diseases, and high yielding. His work greatly promoted the wheat production in the areas of the middle and lower reaches of the Yangtze River.

Lu developed his own theories of plantation, food, nutrition, agricultural production, and population growth, and applied his theories to wheat breeding and agricultural development.

$\mathrm{Lu}$ has published more than 140 research papers and technical reports, on a great variety of issues, as wheat heredity and breeding, crop cultivation techniques, comprehensive development of agriculture of different areas, relationship between progress of technology and modernization of agriculture, and strategy of food development of China.

Lu authored over 10 books, including: The Latest Achievements in Wheat Cultivation Research (1993), The Medium and Long-term Food Development Strategy of China (1993), A General Introduction to the Stereoscopic Agriculture in China (1999), Prospects of Agricultural Development in the Western Areas of China (1999), Food and Nutrition Development in China (2003), Sciences, Technologies, and Development of Agriculture (2003), New Technologies of Modern Agriculture (2006), A General Treatise on the Courtyard Economy in China (2006), Theory and Practice of Agricultural Development of China (2006), Food Safety (2007), and The Anthology of Liang-shu Lu (1999).

Lu loves agriculture and has made great contribution to the development of agriculture of China. His passion, diligence, and creative crop cultivation won him a lot of prizes and honors. His profound knowledge, original ideas, exploring attitudes, and enthusiastic devotions to what he loves inspire younger generations of scientists, technologists and engineers.

$\mathrm{Lu}$ is one of the objects of the case study series of the older generation of scientists as a research project initiated by the Chinese Society of Sciences. The fruit of the study, Inclination to Crop Cultivation - A Biography of Liang-shu Lu, authored by Bin Song and others, was published by Shanghai Jiao Tong University Press in 2013. 